\title{
Annular Plane Systolic Excursion
}

National Cancer Institute

\section{Source}

National Cancer Institute. Annular Plane Systolic Excursion. NCI Thesaurus. Code C139042.

The longitudinal displacement of a cardiac valve annulus toward the apex of the heart. 